\title{
Erratum to: A flow topology optimization method for steady state flow using transient information of flow field solved by lattice Boltzmann method
}

\author{
Kazuo Yonekura ${ }^{1}$ • Yoshihiro Kanno ${ }^{2}$
}

Published online: 20 May 2016

(C) Springer-Verlag Berlin Heidelberg 2016

The original version of this article unfortunately contained mistakes. The aim of this erratum is to correct mistakes of Lemma 1 and claryfy the obscurity of the sentences. In addition, we note that the method works in practice for a nearly self adjoint optimization problems. For non-self adjoint problems the solutions produced by the method are not guaranteed to satisfy first-order necessary optimality conditions.

The first-order necessary conditions for the optimization problem (P) with respect to $\varepsilon$ amd $\boldsymbol{u}$ are written as follows:

$$
\begin{aligned}
\frac{\partial \mathcal{L}}{\partial \varepsilon(t)}= & \frac{\partial D(\boldsymbol{u}, \varepsilon ; T)+C(\boldsymbol{u}, \varepsilon ; T)}{\partial \varepsilon(t)} \\
& -\left\langle\frac{\partial \boldsymbol{u}_{\mathrm{NS}}(\varepsilon, \boldsymbol{x}, t)}{\partial \varepsilon(t)}, \boldsymbol{v}(\boldsymbol{x}, t)\right\rangle+\gamma_{1}(\boldsymbol{x}, t) \\
& -\gamma_{2}(\boldsymbol{x}, t)+\gamma_{3}=0,
\end{aligned}
$$

The online version of the original article can be found at http://dx. doi.org/10.1007/s00158-014-1123-x.

\section{Kazuo Yonekura}

kazuo_yonekura@ihi.co.jp

1 R\&D Technology Center, IHI Corporation, Yokohama, Japan

2 Department of Mathematical Informatics, University of Tokyo, Tokyo, Japan

$$
\begin{aligned}
\frac{\partial \mathcal{L}}{\partial \boldsymbol{u}(t)}= & \frac{\partial D(\boldsymbol{u}, \varepsilon ; T)+C(\boldsymbol{u}, \varepsilon ; T)}{\partial \boldsymbol{u}(t)} \\
& +\left\langle 1-\frac{\partial \boldsymbol{u}_{\mathrm{NS}}(\varepsilon, \boldsymbol{x}, t)}{\partial \boldsymbol{u}(t)}, \boldsymbol{v}(\boldsymbol{x}, t)\right\rangle=0 .
\end{aligned}
$$

We need adjoint equations to solve (E.2). The solution $(\varepsilon, \boldsymbol{u})$ provided by the proposed method does not satisfy (E.1) and (E.2). In a discretized form, the Lagrangian is formulated with using the adjoint variable $\boldsymbol{v}_{0}, \boldsymbol{v}_{1}, \ldots, \boldsymbol{v}_{N}$ as follows:

$$
\begin{aligned}
\mathcal{L}=D\left(\boldsymbol{u}_{N}, \varepsilon_{N}\right) & +C\left(\boldsymbol{u}_{N}, \varepsilon_{N}\right) \\
+ & +\sum_{k=0}^{N}\left(\left\langle\boldsymbol{u}_{k}-\boldsymbol{u}_{\mathrm{LBM}}\left(\boldsymbol{u}_{k-1}, \varepsilon_{k}\right), \boldsymbol{v}_{k}\right\rangle+\left\langle\varepsilon_{k}, \gamma_{1 k}\right\rangle\right. \\
& \left.+\left\langle 1-\varepsilon_{k}, \gamma_{2 k}\right\rangle+\gamma_{3 k}\left(\int_{\Omega} \varepsilon_{k} \mathrm{~d} \boldsymbol{x}-\bar{V}\right)\right)
\end{aligned}
$$

The first-order necessary conditions in the discretized form are written as follows:

$$
\begin{aligned}
\frac{\partial \mathcal{L}}{\partial \varepsilon_{k}}= & \frac{\partial D\left(\boldsymbol{u}_{N}, \varepsilon_{N}\right)+C\left(\boldsymbol{u}_{N}, \varepsilon_{N}\right)}{\partial \varepsilon_{k}} \\
& -\left\langle\frac{\partial \boldsymbol{u}_{\mathrm{LBM}}\left(\boldsymbol{u}_{k-1}, \varepsilon_{k}\right)}{\partial \varepsilon_{k}}, \boldsymbol{v}_{k}\right\rangle \\
& +\gamma_{1 k}-\gamma_{2 k}+\gamma_{3 k}=0
\end{aligned}
$$


$\frac{\partial \mathcal{L}}{\partial \boldsymbol{u}_{k}}= \begin{cases}\frac{\partial D\left(\boldsymbol{u}_{N}, \varepsilon_{N}\right)+C\left(\boldsymbol{u}_{N}, \varepsilon_{N}\right)}{\partial \boldsymbol{u}_{k}}+\boldsymbol{v}_{k}-\left\langle\frac{\partial \boldsymbol{u}_{\mathrm{LBM}}\left(\boldsymbol{u}_{k}, \varepsilon_{k+1}\right)}{\partial \boldsymbol{u}_{k}}, \boldsymbol{v}_{k+1}\right\rangle=0, & \text { if } k<N \\ \frac{\partial D\left(\boldsymbol{u}_{N}, \varepsilon_{N}\right)+C\left(\boldsymbol{u}_{N}, \varepsilon_{N}\right)}{\partial \boldsymbol{u}_{k}}+\boldsymbol{v}_{k}=0, & \text { if } k=N\end{cases}$

In (E.5), the adjoint equations should be employed for $k<$ $N$. It is not necessary only at $k=N$. The sequence $\left\{\varepsilon_{k}\right\}$ and $\left\{\boldsymbol{u}_{k}\right\}$ provided by the proposed method does not satisfy (E.4) and (E.5).

The proposed method solves the following equations by generating sequences $\left\{\varepsilon_{k}\right\}$ and $\left\{\boldsymbol{u}_{k}\right\}$ :

$$
\begin{aligned}
& \frac{\partial D\left(\boldsymbol{u}_{N}, \varepsilon_{N}\right)+C\left(\boldsymbol{u}_{N}, \varepsilon_{N}\right)}{\partial \varepsilon_{N}}-\left\langle\frac{\partial \boldsymbol{u}_{\mathrm{LBM}}\left(\boldsymbol{u}_{k-1}, \varepsilon_{N}\right)}{\partial \varepsilon_{N}}, \boldsymbol{v}_{N}\right\rangle \\
& +\gamma_{1 k}-\gamma_{2 k}+\gamma_{3 k}=0, \\
& \frac{\partial D\left(\boldsymbol{u}_{N}, \varepsilon_{N}\right)+C\left(\boldsymbol{u}_{N}, \varepsilon_{N}\right)}{\partial \boldsymbol{u}_{N}}+\boldsymbol{v}_{N}=0 .
\end{aligned}
$$

The sequences $\left\{\varepsilon_{k}\right\}$ and $\left\{\boldsymbol{u}_{k}\right\}$ are computed by using the gradient of the following function $\mathcal{L}_{k}$ :

$$
\begin{aligned}
\mathcal{L}_{k}= & D\left(\boldsymbol{u}_{k}, \varepsilon_{k}\right)+C\left(\boldsymbol{u}_{k}, \varepsilon_{k}\right)+\left\langle\boldsymbol{u}_{k}-\boldsymbol{u}_{\mathrm{LBM}}\left(\boldsymbol{u}_{k-1}, \varepsilon_{k}\right), \boldsymbol{v}_{k}\right\rangle \\
& +\left\langle\varepsilon_{k}, \gamma_{1 k}\right\rangle+\left\langle 1-\varepsilon_{k}, \gamma_{2 k}\right\rangle+\gamma_{3 k} \int_{\Omega} \varepsilon_{k} \mathrm{~d} \boldsymbol{x} .
\end{aligned}
$$

$\mathcal{L}_{k}$ is the Lagrangian of the following optimization problem $\left(\mathrm{P}_{k}\right)$ for given $\boldsymbol{u}_{k-1}$ :

$$
\begin{array}{ll}
\left(\mathrm{P}_{k}\right) \quad \min _{\varepsilon_{k}, \boldsymbol{u}_{k}} & D\left(\boldsymbol{u}_{k}, \varepsilon_{k}\right)+C\left(\boldsymbol{u}_{k}, \varepsilon_{k}\right), \\
\text { s.t. } & \boldsymbol{u}_{k}=\boldsymbol{u}_{\mathrm{LBM}}\left(\boldsymbol{u}_{k-1}, \varepsilon_{k}\right), \\
& 0 \leq \varepsilon_{k} \leq 1 \\
& \int_{\Omega} \varepsilon_{k} \mathrm{~d} \boldsymbol{x}=\bar{V} .
\end{array}
$$

Therefore, the proposed method can be said to define the sequence of optimization problems $\mathcal{P}=\left\{\left(\mathrm{P}_{0}\right),\left(\mathrm{P}_{1}\right), \ldots,\left(\mathrm{P}_{N}\right)\right\}$ and to approximately trace the path of the optimal solutions of $\mathcal{P}$. The convergence of the sequence $\mathcal{P}$ is not guaranteed. If $\boldsymbol{u}_{k}=\boldsymbol{u}_{\mathrm{LBM}}\left(\boldsymbol{u}_{k-1}, \varepsilon_{k}\right)$ converges, i.e. $\boldsymbol{u}_{N-2}=\boldsymbol{u}_{N-1}=\boldsymbol{u}_{N}$, then the sequence $\mathcal{P}$ converges, i.e. $\left(\mathrm{P}_{N-1}\right)$ is equivalent to $\left(\mathrm{P}_{N}\right)$. Even if $\mathcal{P}$ converges, the optimal solution of $\left(\mathrm{P}_{N}\right)$ does not satisfy the first-order optimality condition of ( $\left.\mathrm{P}_{\mathrm{LBM}}\right)$.

In the following, we correct the mistakes of the original paper. The following sentence at page 160 (right column line 8) was not correct:

In contrast, we propose the optimization algorithm with proved local optimality under certain assumption.

The sentence should be

In the present paper, we apply the method to various types of problems and study the stability of the method.
The following sentence at page 160 (right column line 29) was not correct, and it should be removed:

Hence, adjoint variables are not required, which reduces computational cost.

After equation (11e), the following equation should be added:

$$
\frac{\partial \mathcal{L}}{\partial \boldsymbol{u}}(\boldsymbol{x}, t)=\frac{\partial(D+C)}{\partial \boldsymbol{u}}+\boldsymbol{v}=0 .
$$

Below the sentence including (12), we add the following sentence to clarify the equation:

It should be clear in (12b) that $\boldsymbol{u}$ is considered a variable dependeing on $\varepsilon$ and we obtain $\frac{\partial(D+C)}{\partial \varepsilon}=$ $\left.\frac{\partial(D+C)}{\partial \boldsymbol{u}}\right|_{\varepsilon} \frac{\partial \boldsymbol{u}}{\partial \varepsilon}+\left.\frac{\partial(D+C)}{\partial \varepsilon}\right|_{\boldsymbol{u}}$ by using the chain rule.

Before Lemma 1, the following sentence was not correct.

Nevertheless, the solution of (12) is at least a local optimal solution of $(\mathrm{P})$ at the final stage $(t=T)$ if $\varepsilon$ converges with respect to time. This is shown in the following lemma.

The sentence should be be corrected in order to claryfy the limitation of Lemma 1 as follows:

The following lemma shows that, under some assumptions, (11) is satisfied only at $t=T$, and does not show (11) is satisfied at $t<T$.

The following sentence in the Lemma 1 was incorrect.

If there exists $T^{\prime} \in[0, T]$ such that the solution of (12a) satisfies $\left\|\frac{\partial \varepsilon}{\partial t}\right\| \leq \delta$ for any $\delta>0$ and any $t \in\left[T^{\prime}, T\right]$, i.e., $\varepsilon(t) \rightarrow \varepsilon\left(T^{\prime}\right)=\varepsilon(T)$, then there exists Lagrange multiplier such that $\varepsilon(t)$ satisfies the Karush-Kuhn-Tacker (KKT) conditions.

The sentence should be

If there exists $T^{\prime} \in[0, T]$ such that the solution of (12) satisfies $\left\|\frac{\partial \varepsilon}{\partial t}\right\| \leq \delta$ for any $\delta>0$ and any $t \in\left[T^{\prime}, T\right]$, i.e., $\varepsilon(t) \rightarrow \varepsilon\left(T^{\prime}\right)=\varepsilon(T)$, then there exists Lagrange multiplier such that $\varepsilon(T)$ satisfies (11) at $t=T$.

The following sentence in the proof of Lemma 1 was incorrect. 
Let $\boldsymbol{v}(\boldsymbol{x}, t)=\mathbf{0}$ and $\gamma_{3}=M(T)$, then the left-hand side of (11a) reduces to

$$
\frac{\partial \mathcal{L}}{\partial \varepsilon}(\boldsymbol{x}, t)=\left\{\begin{array}{l}
\gamma_{1}(\boldsymbol{x}, t)-\gamma_{2}(\boldsymbol{x}, t) \text { for } t<T \\
\frac{\partial(D+C)}{\partial \varepsilon}(\boldsymbol{x}, T)+\gamma_{1}(\boldsymbol{x}, t)-\gamma_{2}(\boldsymbol{x}, t)+M(T) \text { for } t=T .
\end{array}\right.
$$

The sentence should be as follows.

Let $\boldsymbol{v}(\boldsymbol{x}, t)=-\left.\frac{\partial(D+C)}{\partial \boldsymbol{u}}\right|_{\varepsilon}$ and $\gamma_{3}=M(T)$, then Eq.

(11f) is satisfied and the left-hand side of (11a) at time $t=T$ reduces to

$$
\begin{aligned}
\frac{\partial \mathcal{L}}{\partial \varepsilon}(\boldsymbol{x}, T)= & \left.\frac{\partial(D+C)}{\partial \varepsilon}(\boldsymbol{x}, T)\right|_{\boldsymbol{u}}-\left\langle\frac{\partial \boldsymbol{u}_{\mathrm{NS}}}{\partial \varepsilon}(\boldsymbol{x}, T), v(\boldsymbol{x}, T)\right\rangle \\
& +\gamma_{1}(\boldsymbol{x}, T)-\gamma_{2}(\boldsymbol{x}, T)+M(T) \\
= & \frac{\partial(D+C)}{\partial \varepsilon}(\boldsymbol{x}, T)+\gamma_{1}(\boldsymbol{x}, T) \\
& -\gamma_{2}(\boldsymbol{x}, T)+M(T),
\end{aligned}
$$

where we use $\frac{\partial(D+C)}{\partial \varepsilon}=\left.\frac{\partial(D+C)}{\partial \boldsymbol{u}}\right|_{\varepsilon} \frac{\partial \boldsymbol{u}}{\partial \varepsilon}+\left.\frac{\partial(D+C)}{\partial \varepsilon}\right|_{\boldsymbol{u}}$ and (12a).

The Case 1 in the proof of Lemma 1 was incorrect and it should be removed.

The following sentence in the Lemma 2 was incorrect.

then there exists Lagrange multiplier such that $\varepsilon(t)$ satisfies the KKT conditions (11) in a discrete form.
The sentence should be

then $\varepsilon_{N}$ satisfies (11) in a discrete form at $k=N$.

The following equation which defines the inlet velocity in section 4.2 was in correctly typeset.
$U_{\text {in2 }}(x)= \begin{cases}-0.5 \bar{U}\left(y-\frac{L}{2}\right)\left(y-\frac{3 L}{4}\right) & \text { for } y \in\left\{y \mid \frac{L}{2} \leq y \leq \frac{3 L}{4}\right\}, \\ 0 & \text { for } y \in\left\{y \mid y \leq \frac{L}{2}, \frac{3 L}{4} \leq y\right\},\end{cases}$

The equation should be as follows.

$U_{\operatorname{in} 2}(x)= \begin{cases}-0.5 \bar{U}\left(x-\frac{L}{2}\right)\left(x-\frac{3 L}{4}\right) & \text { for } x \in\left\{x \mid \frac{L}{2} \leq x \leq \frac{3 L}{4}\right\}, \\ 0 & \text { for } x \in\left\{x \mid x \leq \frac{L}{2}, \frac{3 L}{4} \leq x\right\},\end{cases}$

The following sentence at page 171 (right column line 15) was not correct:

Also, it does not need to solve adjoint equations.

The sentence should be

Also, it does not solve adjoint equations.

The following sentence at page 171 (right column line 17) was not correct and it should be removed

Moreover, if the method converges, then the obtained solution is guaranteed to be at least a local optimal solution.

Acknowledgments We express our gratitude to Dr. Evgrafov for pointing out the mistakes. 